\title{
Safety of single incision laparoscopic cholecystectomy using Gloveport and easy conversion technique to one additional port
}

\author{
Sung Yub JEONG ${ }^{1}$, Sung Hwan LEE ${ }^{2}$, Sung Hoon $\mathrm{CHOI}^{* 2}$ \\ 'Department of General Surgery, Armed Forces Capital Hospital, Seongnam, Korea \\ ${ }^{2}$ Division of Hepatobiliary and Pancreas, Department of Surgery, CHA Bundang Medical Center, CHA University, Seongnam, Korea
}

Introduction: Our center successfully incorporated the alignment of the instruments in robotic single-site cholecystectomy into single incision laparoscopic cholecystectomy (SILC). Since then, SILC has been applied to almost all cases involving gallbladder except for malignancies. SILC can also be applied to complicated cases with the use of additional port as a safe alternative. We introduce our experience of implementing easy conversion of one additional port during SILC.

Methods: Between March 2014 and December 2020, 1,126 patients underwent SILC in our center by 3 hepatobiliary surgeons. The indication included benign diseases of gallbladder. When conversion was needed, one additional port was inserted at subxiphoid area. Perioperative outcomes were reviewed.

Results: 440 males and 686 females were included in this study. The mean age was $47.9 \pm 13.4$ years, mean body mass index was $24.6 \pm$ $3.6 \mathrm{~kg} / \mathrm{m}^{2}$, and mean operative time was $47.6 \pm 17.3$ minutes. Total number of 67 patients $(6.0 \%)$ were required to one additional port, of which 41 patients due to Calot's triangle adhesion, 14 patients due to uncontrolled bleeding at Calot's triangle, 9 patients due to severe inflammation, and 3 patients due to poor operation field.

Conclusions: Based on our vast number of cases of SILC no matter how complicated the case, one subxiphoid additional port can be a safeguard to SILC. Therefore, bear in mind SILC can be endeavored by all surgeons with the use of additional port as a safe alternate. 